\title{
Birth of a Healthy baby from Abdominal Pregnancy, A Rare Event: A Case Report
}

\author{
Nahreen Akhtar', Firoza Begum ${ }^{1}$, Tabassum Parveen ${ }^{2}$, Syeda Sayeeda ${ }^{3}$, Sabina Karim ${ }^{4}$ \\ 'Professor, ${ }^{2}$ Associate Professor, Fetomaternal Medicine Wing, Department of Obs \& Gynac, BSMMU, ${ }^{3}$ Associatc Professor, Department of Obs \& \\ Gynae, BSMMU, ${ }^{4}$ MD Residency, Paediatric Haematology \& Oncology, BSMMU.
}

\begin{abstract}
:
Abdominal pregnancy is a rare form of ectopic pregnancy with very high morbidity and mortality for both the mother and the fetus. Diagnosis and management can pose some difficulties especially in low resource centres. High index of suspicious is vital in making prompt diagnosis in such situations. A young lady of 30 years presented with 2 nd gravida at 31 wks pregnancy with pain in the whole abdomen having loose motion admitted in Dept of Obs and Gynae BSMMU. She was diagnosed as a case of 31 wks pregnancy with gastroenteritis. She was treated conservatively according to advice of gastroenterologist and was discharged after improvement. Patient was readmitted again with pervaginal watery discharge. Decision for cesarean section was taken because of transverse lie, fetal growth restriction with severe oligohydramnios. After opening the abdomen, abdominal pregnancy was diagnosed. Following delivery of the baby significant bleeding started which was secured with ligature. Placenta was kept in situ. Mother and baby were discharged healthy on fourteenth postoperative day.
\end{abstract}

Key words: abdominal pregnancy, pregnancy, haemorrhage

\section{Introduction:}

An abdominal pregnancy is a form of an ectopic pregnancy where the pregnancy is implanted within the peritoneal cavity, outside the fallopian tube or ovary and not located in the broad ligament. 1 While rare, abdominal pregnancies have a higher mortality rate than ectopic pregnancies in general but, on occasion, can lead to a delivery of a viable infant. Less than $1 \%$ of ectopic pregnancies in the United States are abdominal, or about 1 out of every 10,000 pregnancies.' Risk factors are similar to tubal pregnancy with sexually transmitted disease playing a major role. ${ }^{2}$ The maternal mortality rate is estimated to be about 5 per 1,000 cases, about seven times the rate for ectopics in general and about 90 times the rate for a delivery. $1 \mathrm{~A}$ patient with an abdominal pregnancy may just display the normal signs of pregnancy or have non-specific symptoms such as abdominal pain, vaginal bleeding, and/or gastrointestinal symptoms. ${ }^{3}$ Frequently

Corresponding Author: Professor Dr. Nahreen Akhtar

Fetomatemal Medicine Wing, Department of Obs \& Gynae, BSMMU.

E mail- nahreenaklitar10@gmail.com , Ph- 01711544687.
[BSMMU J 2016; 9 (1) : 71-74]

the diagnosis of an abdominal pregnancy is missed. ${ }^{4}$ Suspicion of an abdominal pregnancy is raised when the baby's parts can be easily felt, or the lie is abnormal. Sonography is extremely helpful in the diagnosis as it can demonstrate that the pregnancy is outside an empty uterus, there is no amniotic fluid between the placenta and the fetus, no uterine wall surrounding the fetus, fetal parts are close to the abdominal wall, and the fetus is in abnormal lic. ${ }^{2}$ MRI has also been used with success to diagnose abdominal pregnancy. ${ }^{5}$ Elevated alpha-fetoprotein levels are another clue of the presence of an abdominal pregnancy. ${ }^{6}$ Abdominal pregnancy at term with a healthy viable fetus is therefore an extremely rare condition and very few of such cases have been published during the last ten years. We present a case of abdominal pregnancy that resulted in a term live baby without malformations.

\section{Case Report:}

A 30 years old woman, Para 0+1 ectopic pregnancy, was referred from a private clinic on 1st Dec 2013. Her principal complaint on arrival was 31 wks pregnancy with per 
vaginal watery discharge. She is married for $10 \mathrm{yrs}$ and had secondary subfertility. She had right sided salpingectomy for ectopic pregnancy 8 yrs back. She had regular menstrual cycles prior to her pregnancy. Her last menstrual period was on 20.4.13 and accordingly her Expected date of delivery will be on 27.1.14. Her 1st trimester was incident free. During 2nd trimester at her 28wks she suffered from upper abdominal pain which kept her out of work. At the same time she had vomiting, loose motion several times and got admitted in Dept of Obs \& Gynae, BSMMU on 3rd November 2013. At that time on per abdominal examination, abdomen was found slightly distended and diffuse tenderness. Her height of uterus was not well defined. She was treated conservatively by tiemonium and I/V fluid. Consultation with gastroenterologists was taken for loose motion and suggested to keep her nothing by mouth for 5 days, followed by liquid, soft and subsequently by normal diet. She was treated by $I / V$ fluid, Inj antiulcerant and Inj Ketorolac for pain abdomen. With these management abdomen became soft and patient was improved. Then all the drugs were given orally. She was anemic ( $8 \mathrm{gm} \%$ ) and 2 units of blood was transfused. Ultrasonography revealed 28 wks pregnancy with fetal growth restriction with severe oligohydramnios with soft tissue lesion in the lower abdomen. Her liver function tests were normal, but her serum amylase was slightly raised, 95IU/L and serum lipase level was $707 \mathrm{U} / \mathrm{L}$. She was discharged on 16th November 2013 with advice for regular antenatal check up. She was however, again kept out of work during the third trimester for abdominal pain. Her antenatal card revealed nine visits and a gestational age of 32 weeks at the time of referral. On examination, she looked hemodynamically stable. She was mildly anemic. Cardiovascular and respiratory systems examinations did not reveal any abnormalities. Her abdominal examination revealed symphysio-fundal height was $31 \mathrm{~cm}$, transverse lie, foetal heart rate of 136 beats per minute and there was no uterine contractions, although the fundal height was not well defined. Vaginal examination was not done. She had five ultrasound scan examination with the last two within seven days of admission indicating intrauterine gestation with transverse lie. The rest of her investigations were normal. The haemoglobin level was $10.9 \mathrm{~g} / \mathrm{dl}$ and blood group was $O$ Rhesus positive. She was booked for caesarean section on account of transverse lie with severe oligo- hydramnios. Pfannensteil incision was given. At laparotomy on opening the abdominal cavity uterus is not seen rather a sac like structure was noted with which gut were found to be attached. Giving a small incision on the sac like area baby was seen without any liquor and a live female baby weighing 1.5 kilograms was taken out. On exploring the pelvic cavity uterus was found almost of normal size and right ovary and tube looked healthy. Left tube and ovary was not identified as it was removed earlier for ectopic pregnancy. Placenta and cord was noted to be morbidly adherent with the surrounding gut and omentum. Huge bleeding started from the separated sites of the placenta which were catched and ligated as far as possible, but bleeding continued and site of bleeding was difficult to control. With surgical consultation bleeding point was ligated. Placenta was left in situ. Total estimated peroperative bleeding was one and half litre. Three units of compatible blood was transfused per and postoperatively. Abdomen was closed keeping a drain tube. She was transferred to ICU because of hemodynamically unstable. Inj Ceftriaxone, Gentamycin and Metronidazole was given. She was returned to Obstetric ward on Ist postoperative day. Her $\mathrm{Hb} \%$ was $8.6 \mathrm{gm} / \mathrm{dl}$ on 2 nd POD. Reports of serum electrolyte, blood sugar and serum creatinine were normal. Rest of her postoperative period was uneventful. Her baby was managed in neonatology ward because of low birth weight. The patient progressed well and was finally discharged home on fourteenth postoperative day. She was followed up weekly for four weeks by serum $\beta$ HCG. Abdominal ultrasound was done after six weeks and showed normal size uterus and ovaries and the portion of placenta that was left in situ was not identified. Beta human chorionic gonadotropin ( $\mathrm{BhCG}$ ) was negative at the same period. The baby was also found healthy at 6 weeks of postnatal period.

\section{Discussion:}

Advanced abdominal pregnancy refers to situations where the pregnancy continues past 20 weeks of gestation. ${ }^{7}$ In a report from Nigeria four live births out of a series of 20 abdominal pregnancies have been reported. ${ }^{8}$ Often, however, with advancement of the pregnancy the support for the fetus becomes compromised and the fetus dies. A patient may carry a dead fetus but will not go into labor. Over time, the fetus calcified and becomes a lithopedion. 
It is generally recommended to perform a laparotomy when the diagnosis of an abdominal pregnancy is made. ${ }^{3}$ However, if the pregnancy is past 24 weeks and the baby alive and medical support systems are in place, careful watching could be considered to bring the baby to viability (34-36 weeks). ${ }^{3}$ Women with an abdominal pregnancy will not go into labor. Delivery in a case of an advanced abdominal pregnancy will have to be via laparotomy. The survival of the baby is reduced and high perinatal mortality rates between $40-95 \%$ have been reported. ${ }^{9}$ Babies of abdominal pregnancies often have birth defects due to compression in the absence of the amniotic fluid buffer. The rate of malformations and deformations is estimated to be about $21 \%$; typical deformations are facial and cranial asymmetries and joint abnormalities and the most common malformations are limb defects and central nervous malformations. ${ }^{10}$ Once the baby has been delivered placental management becomes an issue. In normal deliveries the contraction of uterus provides a powerful mechanism to control blood loss, however, in an abdominal pregnancy the placenta is located over tissue that cannot contract and attempts of its removal may lead to significant blood loss. Generally, unless the placenta can be easily tied off or removed, it may be preferable to leave it in place and allow for a natural regression. ${ }^{2.3}$ This process may take about four months and can be monitored by checking human chorionic gonadotropin levels. Use of methotrexate to accelerate placental regression is controversial as the large amount of necrotic tissue is a potential site for infection. ${ }^{2}$ Placental vessels have also been blocked by angiographic embolization."

Advanced abdominal pregnancy is extremely rare. In a review at the Komfo Anokye Teaching Hospital, OpareAddo et al reported an incidence of 1:1320 deliveries ${ }^{12}$ whilst Amirtha et al cited 1:25000 deliveries. ${ }^{13}$ Most of the cases of abdominal pregnancies are secondary from aborted or ruptured tubal pregnancy. ${ }^{13}$ In this case it was obvious that the abdominal implantation was secondary to undiagnosed ruptured left tubal ectopic pregnancy. Clinical diagnosis can be very difficult and ultrasound is very helpful during the early stages of gestation but can also be disappointing in the later stages.

Other radiological studies such as MRI and CT scan are helpful in the later stages. ${ }^{14}$ Teng et al reported an interesting case in which MRI played a decisive role in the diagnosis ${ }^{15}$, unfortunately these advanced imaging technologies are not available in most parts of the third world. Our patient had five ultrasound scan examinations and none of this suggested the possibility of abdominal pregnancy. In poorly resourced centres, high index of suspicion is key for prompt diagnosis and timely intervention to prevent life-threatening complications.

Bleeding from placental implantation site is the most life-threatening complication during laparotomy. The decision to remove the placenta or not can be a determining factor for the survival or otherwise of the woman and this decision is subject to the surgeon's expertise and the particular case in question. It is generally recommended to leave the placenta in situ and make a follow up with human chorionic gonadotropin levels. ${ }^{16}$ In this case there was significant bleeding from some detached portions of the placenta that prompted removal of these portions to secure haemostasis. The patient was transfused with three unit of blood during the operation and that was enough. For the newborn, it is very important to rule out congenital malformations. There are reports of fetal malformations as high as $40 \%$ associated with abdominal pregnancies and only $50 \%$ of these babies survive up to one week post delivery. ${ }^{17,18}$ In his extensive review, Stevens found some varying degrees of deformations and malformations in $21.4 \%$ of these infants. ${ }^{19}$ In this case that has been presented, no malformation has been found on the child after birth.

\section{Conclusions:}

Abdominal pregnancy with resultant healthy newborn is very rare. Diagnosis of the condition can be difficult especially if the pregnancy is advanced. High level of suspicion, careful clinical and ultrasound examinations are the routine means of diagnosis though $\mathrm{CT}$ scan and MRI can be useful. Bleeding is the single most important life-threatening complication for the mother whilst fetal malformation is one of the numerous challenges that can 
confiont the newborn.

\section{References:}

01. Atrash HK, Friede A, Hogue CJR. "Abdominal Pregnancy in the United States: Frequency and Mortality". Obstetrics and Gynecology $1987 ; 63(3): 333-7$

02. Kun KY, Wong PY, Ho MW, Tai CM, Ng TK. "Abdominal pregnancy presenting as a missed abortion at 16 weeks' gestation". Hong Kong Medical Joumal 2000;6 (4): 425-7.

03. Maurice King, Peter C. Bewes, James Cairns, Jim Thomton (editors). "Primary Surgery; Volume One: Non-trauma. Chapter 8 , Abdominal pregnancy". Bonn University. Retrieved 2010-01-25.

04. Adeoye I, Twomey D, Egwuatu EV, Okonta PI. "A 30-year review of advanced abdominal pregnancy at the Mater Misericordiae Hospital, Afikpo, southeastem Nigeria (1976-2006)". Archives of Gynecology and Obstetrics 2011;283 (1): 19-24.

05. Dahiya K, Sharrna D. "Advanced Abdoninal Pregnancy: A Diagnostic and Management Dilemma". Joumal of Gynecologic Surgery $2007 ; 23$ (2): 69-72.

06. Tromans PM, Coulson R, Lobb MO, Abdulla U. Abdominal pregnancy associated with extremely elevated serum alpha fetoprotein: case report. British Journal of Obstetrics and Gynaecology $1984 ; 91$ (3): 296-8.

07. White RG. Advance Abdominal Pregnancy - A Review of 23 Cases. Irish Journal of Medical Science $1989 ; 158$ (3) : 77-8.

08. Sunday A I, Twomey D, Egwuatu EV, Okonta PI. "A 30-year review of advanced abdominal pregnancy at the Mater Misericordiae Hospital, Afikpo, southeastem Nigeria (1976-2006)". Archives of Gynecology and Obstetrics 2011;283 (1): 19-24

09. Martin JN Jr, Sessums JK, Martin RW, Pryor JA, Morrison JC "Abdominal pregnancy: current concepts of management".
Obstetrics and Gynecology 1988;71 (4): 549-57.

10. Stevens CA. "Malformations and deformations in abdominal pregnancy". American Journal of Medical Genetics 1993;47 (8): 1189-95.

I1. Cardosi RJ, Nackley AC, Londono J, Hoffman M. "Embolization for advanced abdominal pregnancy with a retained placenta. A case report". Reproductive Medicine 2002; 47 (10): 861-3.

12. Opare A HS, Daganus S. Advanced abdominal pregnancy: a study of 13 consecutive cases seen in 1993 and 1994 at Komfo Anokye Teaching Hospital, Kumasi, Ghana. Afr J Reproductive Health 2000; 4(1): 28-39. [PubMed]

13. Amritha B, Sumangali T, Priya B, Deepak S, Rai S. A rare case of term viable secondary abdominal pregnancy following rupture of a rudimentary hom. A case report. J Med case reports 2009; 3:38. [PubMed]

14. Karat LS. Viable Abdominal Pregnancy. J Obstet Gynecol 2007; $57(2): 169-170$

15. Cunningham F, Gant N, Leveno K. Ectopic Pregnancy. Williams Obstetrics. Mcgraw-Hill; 2001; pp. 899-902.

16. Jianping Z, Fen L, Qiu S. Full-Term Abdominal Pregnancy. A Case Report and Review of the Literature. Gynecol Obstet Invest 2008 ; 65(2): 139-141.

17. Teng H, Kumar G, Ramli N. A viable secondary intra-abdominal pregnancy resulting from rupture of uterine scar: role of MRI. Br J Radiol 2007; 80:134-136.

18. Kun K, Wong P, Ho M, Tai C. Abdominal pregnancy presenting as a missed abortion at 16 weeks gestation. Hong Kong Med J $2000 ; 6(4): 425-427$.

19. Stevens CA. Malformations and deformations in abdominal pregnancy. Am J Med Genet 1993; 47(8):1189-1195. 